\title{
Microstructure, Mechanical Properties and Texture Evolution of Mg-Al-Zn-La-Gd-Y Magnesium Alloy by Hot Extrusion and Multi-Pass Rolling
}

\author{
Qiyu Liao ${ }^{1} \cdot$ Wenxin $\mathrm{Hu}^{2} \cdot$ Qichi Le ${ }^{1} \cdot$ Xingrui Chen ${ }^{1} \cdot \mathrm{Ke} \mathrm{Hu}^{1} \cdot$ Chunlong Cheng ${ }^{1} \cdot$ Chenglu Hu${ }^{1}$
}

Received: 2 February 2020 / Revised: 24 February 2020 / Published online: 14 May 2020

(c) The Chinese Society for Metals (CSM) and Springer-Verlag GmbH Germany, part of Springer Nature 2020

\begin{abstract}
A high-ductility $\mathrm{Mg}-8.10 \mathrm{Al}-0.42 \mathrm{Zn}-0.51 \mathrm{Mn}-1.52 \mathrm{La}-1.10 \mathrm{Gd}-0.86 \mathrm{Y}$ (wt\%) alloy was developed by hot extrusion and multi-rolling processes. Relationships between microstructure, mechanical properties and texture evolution of the extruded and rolled alloy were investigated. The rolling process had significant effect on grain refinement of the extruded plate. The grain size reduced from 12.3 to $4.9 \mu \mathrm{m}$ with the increasing rolling pass. With the increase in rolling pass, the proportion of dynamic recrystallized (DRXed) grains increases due to particle-stimulated nucleation, grain boundary nucleation and twin induced nucleation. In the process of multiple rolling, the basal pole gradually tilted from normal direction to transverse direction due to the asymmetric deformation and irregular grain deformation, resulting in the weakening of the base texture. The results showed that grain refinement and texture weakening were the main reasons for the good ductility of the alloy.
\end{abstract}

Keywords Extrusion $\cdot$ Multi-rolling $\cdot$ Grain refinement $\cdot$ Texture evolution $\cdot$ Mechanical properties

\section{Introduction}

Wrought magnesium alloy has huge application potential in aspects of military, aeronautics and astronautics and civil, etc., due to quite a lot excellent characteristics, such as specific stiffness, superior specific strength, good damping property and light weight [1-3]. Mg-Al-Zn systemic wrought magnesium alloys are widely used in industrial products. Nevertheless, the applications are limited because of their strong anisotropy and poor formability at room temperature due to its hexagonal close packed (HCP) structure [3]. It is widely known that excellent formability and ductility during hot deformation process can be achieved via grain refinement and texture weakening. Miao et al. [4] illustrated grain size reduction from $25-40$ to $2.8-4 \mu \mathrm{m}$ and remarkable

Available online at http://link.springer.com/journal/40195.

Qichi Le

qichil@mail.neu.edu.cn

1 Key Lab of Electromagnetic Processing of Materials, Ministry of Education, Northeastern University, Shenyang 110819, China

2 Baotou Research Institute of Rare Earths, Baotou 014000, China ductility of 30-35\% in fracture elongation via the three-pass conventional rolling process. Wang et al. [5] showed that the fracture elongation was significantly improved and the grain size was obviously refined due to the weakness of the $\{0002\}$ basal texture via five passes rolling deformation of ZK60 magnesium alloy. Dynamic recrystallization (DRX) in the multi-rolling process has significant influence on grain refinement, ductility enhancing and texture ameliorating [6].

In general, extruded plate or rolled sheet of magnesium alloy exhibits typical basal texture with majority of c-axis parallel to the extruded or rolled plane [7]. For instance, a typical $<10 \overline{1} 0>$ fiber texture may be established in the extrusion process, which can be transferred to $<10 \overline{1}$ $0>-<11 \overline{2} 0>$ fiber texture. This texture transformation aligns the basal planes parallel to the extrusion direction (ED) [8]. As anisotropy in rolled sheets is ineluctable especially in wrought $\mathrm{Mg}$ alloys, the mechanical property inhomogeneity usually occurs in the products, which is not benefit to widespread applications. Alloying with some rare earth elements, such as $\mathrm{La}, \mathrm{Gd}, \mathrm{Ce}$, and so on, can obvious weak the basal texture of $\mathrm{Mg}-\mathrm{Al}-\mathrm{Zn}$ systemic alloy [9]. The basal pole may tilt from normal direction (ND) to transverse direction (TD) during multi-pass rolling processes, which is commonly favorable for the ductility improvement of wrought $\mathrm{Mg}$ alloys. Kim et al. [10] reported that the randomization 
of basal texture concentrates on TD after fifth-pass rolling processes.

Generally, flat billets are prepared for the following-up rolling process, while round billets are prepared for extrusion deformation. It is well known that it is difficult to manufacture magnesium alloy billet in cast form, while it is easy to manufacture round billet. In addition, it is difficult to product magnesium alloy sheet via extrusion deformation. However, it is easy to produce high-quality sheets by rolling. Therefore, the method of preparing magnesium alloy sheet by extrusion and rolling is worth studying.

Up to now, a lot of studies have been made on the evolution of microstructure and mechanical properties of magnesium alloys during the multi-pass rolling. In general, strong basal texture is usually achieved in the extruded magnesium alloy, which is not conducive to subsequent application. In the present paper, we endeavor to improve the quality of sheet products suitable for industry requirement via exploiting more efficient deformation process. Nucleation mechanism of DRXed grains, texture evolution and ductility improvement during the hot extrusion and following multirolling processes for $\mathrm{Mg}-\mathrm{Al}-\mathrm{Zn}-\mathrm{La}-\mathrm{Gd}-\mathrm{Y}$ alloy were systematically studied in this paper.

\section{Material and Experimental Procedures}

The magnesium alloy studied in this work is traditional AZ80 magnesium alloy with addition of a small quantity of $\mathrm{La}, \mathrm{Gd}$ and Y elements. The chemical composition (wt\%) of the modified AZ80 magnesium alloy is listed in Table 1. The high-purity (99.95\%) Mg, Mg-30\% Gd, Mg-30\% La and $\mathrm{Mg}-30 \% \mathrm{Y}$ (wt\%) master alloys were prepared to melt in high-temperature resistance furnace at 973-1013 K. The round ingot with diameter of $330 \mathrm{~mm}$ was produced by using a low-frequency electromagnetic semi-continuous system. The ingot was homogenized at $693 \mathrm{~K}$ for $24 \mathrm{~h}$ and cooled in the air. Then, the $\varnothing 330 \mathrm{~mm}$ ingot was turned to $\varnothing 300 \mathrm{~mm}$ round ingot in a lathe in order to cooperate with the $3000 \mathrm{t}$ horizontal extruder in the following extrusion process. Subsequently, the ingot and extrusion die were reheated at $603 \mathrm{~K}$ for $30 \mathrm{~min}$. The extrusion process was carried with extrusion rate of $1.5 \mathrm{~mm} / \mathrm{s}$ and extrusion ratio of 18.3 using an extrusion die with cross-sectional dimensions of $300 \mathrm{~mm} \times 20 \mathrm{~mm}$. The extrusion plate was then divided into four samples with length of $100 \mathrm{~mm}$ along the ED for investigation. The extrusion plates were reheated at $623 \mathrm{~K}$ for $30 \mathrm{~min}$ and then rolled on a four stand tandem mill at a speed of $0.3 \mathrm{~m} / \mathrm{s}$. Rolling conditions and rolling processes are given in Table 2. The thickness of the extruded plate was reduced from 20 to $17.3 \mathrm{~mm}$ during the first rolling process, a reduction of about $13.5 \%$. Then, another extruded plate was heated at $623 \mathrm{~K}$ for $10 \mathrm{~min}$, and after two pass rolling process, the thickness of the rolled sheet was decreased to $13.4 \mathrm{~mm}$. The three-pass and four-pass rolling processes were as the same way, and the thickness of the rolled sheets totally reduced $23.6 \%$ and $20.9 \%$, respectively. All the plates were cooled in the air after thermal deformation. Figure 1 shows the process flow diagram of hot extrusion and multipass hot rolling.

Microstructure characteristics of the extruded and rolled specimens were investigated in the ED-ND and RD (rolling direction)-ND plane. The samples for investigated were cut out to $10 \mathrm{~mm}$ in length along the ED/RD and $10 \mathrm{~mm}$ in width along the ND at the middle of the plates. In addition, further observation analysis was performed by the abovementioned SEM equipped with 9 EDAX/TSL electron backscatter diffraction (EBSD). In order to obtain better EBSD results, multi-function Ion Milling surface was used for ion thinning, with $6.5 \mathrm{kV}$ working voltage and 35 min working time. Orientation imaging microscopy (OIM) software was used to analyze EBSD data. The sample was made into the transmission electron microscope sample with a diameter of $\varphi 3 \mathrm{~mm}$ and a thickness of $30 \mu \mathrm{m}$, and the micro-analysis was performed at $200 \mathrm{kV}$ using a transmission electron microscope (TEM) of a JEOL JEM-F200 TEM. The
Table 1 Chemical composition (wt $\%$ ) of the modified AZ80 magnesium alloy

Table 2 Rolling conditions and rolling parameter of the extrusion plates

\begin{tabular}{lllllll}
\hline Al & Zn & Mn & La & Gd & Y & Mg \\
\hline 8.10 & 0.42 & 0.51 & 1.52 & 1.10 & 0.86 & Bal. \\
\hline
\end{tabular}

\begin{tabular}{lllll}
\hline $\begin{array}{l}\text { Pass } \\
\text { tion }\end{array}$ & $\begin{array}{l}\text { Rolling direc- } \\
\text { Temperature }(\mathrm{K})\end{array}$ & Thickness change $(\mathrm{mm})$ & Thickness reduction (\%) \\
\hline 0 & - & $603 \pm 5$ & $\emptyset 300-300 \times 20$ & Primary extrusion \\
1 & IED & $623 \pm 5$ & $20-17.3$ & 13.5 \\
2 & IED & $623 \pm 5$ & $20-17.3-13.4$ & $13.5+22.5$ \\
3 & IED & $623 \pm 5$ & $20-17.3-13.4-10.2$ & $13.5+22.5+23.6$ \\
4 & IED & $623 \pm 5$ & $20-17.3-13.4-10.2-8.3$ & $13.5+22.5+23.6+20.9$ \\
\hline
\end{tabular}




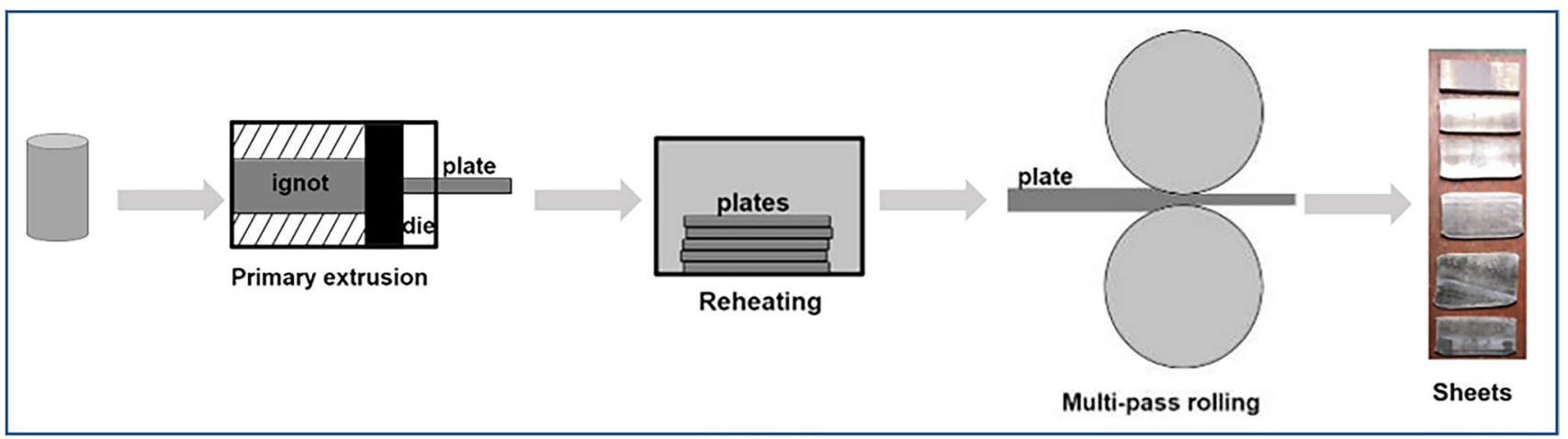

Fig. 1 Process flow diagram of the hot extrusion plate and multi-pass hot rolling sheets

texture analysis was carried by applying EBSD test. The pole figures were measured contained three crystallographic planes $\{0002\},\{10 \overline{1} 0\}$ and $\{10 \overline{2} 0\}$. TEM was used to image microscopic features. The mechanical properties of the extrusion plate and rolling sheets were tested at room temperature using a SANS CMT-5105 material tensile prototype at a strain rate of $10^{-3} \mathrm{~s}^{-1}$. The tensile tested samples were cut out along the ED/RD. The total length of the tensile specimen is $100 \mathrm{~mm}$, the gauge distance of tensile specimen is $40 \mathrm{~mm}$, and the thickness is $2 \mathrm{~mm}$.

\section{Results}

\subsection{Microstructure Characteristics}

The microstructures of the extruded plate in the ED-ND plane are shown in Fig. 2. Figure 2a, c shows bimodal microstructure with an average grain size of $12.3 \mu \mathrm{m}$. The refined equiaxed grains are emerged by DRX. Some coarse grains with diameter of 15-25 $\mu \mathrm{m}$ and fine grains with diameter of $2-15 \mu \mathrm{m}$ are shown in the extruded sample. The broken second phase and fine grain together constitute the deformation band along the ED. The fine DRXed grains locate inside the deformation band can be considered as deformation band dynamic recrystallized (dbDRXed) grains. Figure $2 b$ illustrates inverse pole figure (IPF) and grain misorientation distribution of the extruded sample. The grains with misorientation angle between $5^{\circ}$ and $15^{\circ}$ are regarded as MAGBs (middle-angle grain boundaries). The LAGBs (lowangle grain boundaries) misorientation angle is less than $5^{\circ}$. Most of grains shape with LAGBs, and the density of MAGBs is particularly low. Amounts of LAGBs structures transform to HAGBs (high-angle grain boundaries) during extrusion process.

The microstructure characteristics of the rolled sheets (RD-ND plane) are shown in Fig. 3. It can be observed in Fig. 3a-d that typical bimodal microstructure is exhibited in the rolled AZ80RE alloys. As we can see in Fig. 3i, after the first pass rolling process, the average grain size decreases to $10.3 \mu \mathrm{m}$ and the SD value increases to 8.1 , which is slightly larger than the extruded sample. Part of new DRXed grains are surround the coarse grains, a so-called necklace structure, which can be regarded as nDRXed grains. The average grain size decreases to $7.4 \mu \mathrm{m}$ and the SD value decreases to 6.3 of the second pass rolling sample. From Fig. 3b, j, the area fraction of fine grains with an average diameter of less than $5 \mu \mathrm{m}$ increases compared with the first pass rolling. The average grain size slightly decreases to $6.9 \mu \mathrm{m}$ after the third pass rolling process. The nDRXed grains form the structure of the third pass rolled grains. The average size decreases to $4.9 \mu \mathrm{m}$ and the SD value decreases to 3.5 during the fourth pass rolling process. From Fig. 3c, d, fully DRX occurs after the third and fourth pass rolling. It illustrates that with the increase in rolling pass, the degree of DRX increases. The increase in deformation is conducive to the introduction of high-density dislocation in the material and the nucleation of DRX. Meanwhile, from Fig. 3e-h, the misorientation angle of the rolled samples is mainly concentrated in LAGBs and HAGBs, and the distribution proportion of MAGBs is low. With the increase in rolling pass, amounts of LAGBs convert into HAGBs. It can be seen in Fig. $3 \mathrm{~g}$ that the fine grains homogeneously distribute in the fourth pass rolled sample. Nucleation of fine grains is usually caused by highdensity dislocations. The main reason is that $\mathrm{Gd}$, La and $\mathrm{Y}$ elements in the matrix reduce stacking fault energy and dislocation cross-slip ability, which is more conducive to the nucleation of DRX grains [11]. Therefore, particles in the matrix provide more possibilities for DRX nucleation. Meanwhile, these particles also hinder the grain boundary slipping, which is beneficial to the grain refinement of the extruded plate and rolled sheets [12]. The activation energy of plastic flow increases to a value close to the activation energy for magnesium self-diffusion, and the controlling process is dislocation climb [13]. 

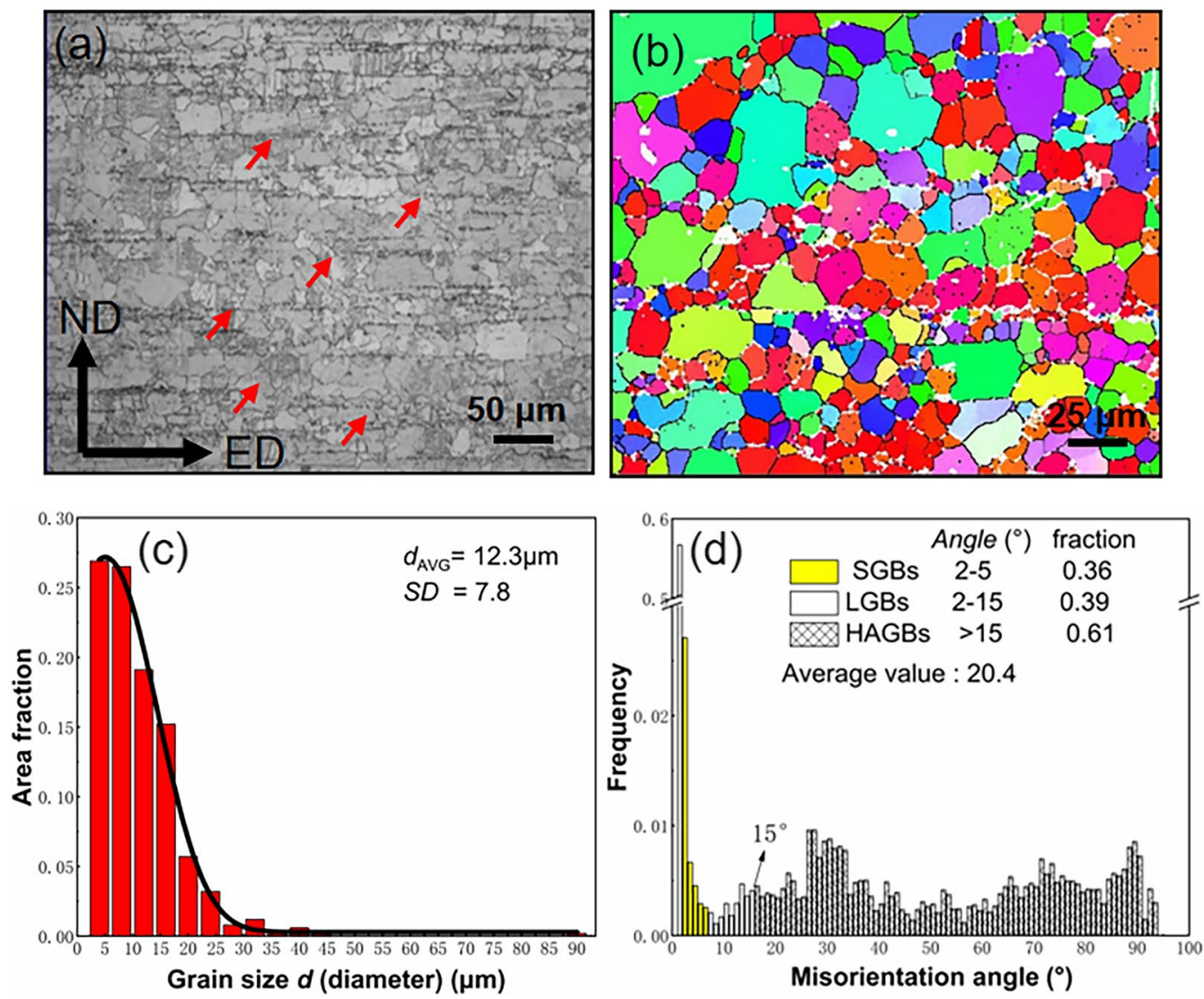

Fig. 2 Microstructure characteristics of the extruded sample of the alloy (ED-ND plane): a optical morphology map; $\mathbf{b}$ IPF (inverse pole figure) map; c average grain size versus area fraction distributions map and the black curve means lognormal fitting of the grain size distributions; d grain misorientation distribution map; $\left(d_{\mathrm{AVG}}\right.$ : the diameter of average grain size; SD: standard deviation of grain size)

\subsection{Texture}

Texture intensity, grain rotation and orientation characteristics are critical in analysis of mechanical properties of magnesium alloys sheets, which can be tested by EBSD test. The $\{0001\},\{10 \overline{1} 0\}$ and $\{1 \overline{2} 10\}$ pole figures of the alloy during extrusion and multi-rolling processes are displayed in Fig. 4. Figure 5 shows the schematic diagram of grain rotation characteristics in the ED/RD-ND plane. As shown in Fig. 4a, after the extrusion process, a typical $\{0001\}$ fiber basal texture is established in the ED-ND plane. Apparently, the fiber components are not concentrated in some particular regions, whose $\{0001\}$ texture intensity spreads along the ND. However, owning to the large deformation, the $c$-axis of most initial grains are parallel to TD or parallel to ND in the $\{0001\}$ plane. In the process of large extrusion deformation, due to the thickness of the plate, the alloy is affected by asymmetric extrusion deformation, resulting in different deformation strains and irregular grain deformation [14].

Compared with the extruded samples, the texture strength of the multi-pass rolled samples decreased gradually (seen in Fig. 4b-e). After the first pass rolling, the
$\{0001\}$ basal texture intensity decreases and the maximal intensity region along ND has an inclination angle of approximately $30^{\circ}$. The basal texture displays doublepeak distribution. Moreover, compared with the extruded samples, the majority of grains still maintain the initial orientation during the first pass rolling. After the next rolling pass, the grain orientation is in a certain direction, and the inclination angle of the $c$-axis is about $30^{\circ}, 60^{\circ}$ and $80^{\circ}$. After the third pass rolling, it is worth noting that the grains parallel to ND tend to disappear and the regions with the maximum base texture tend to be concentrated in ND (seen in Fig. 4d). After the fourth pass rolling, the $\{0001\}$ basal texture of the selected grains almost disappears and the c-axis is parallel to ND. The grain rotation processes during the extrusion and multi-pass rolling deformation are shown in Fig. 5. According to the research of Huang et al. [15], the rolling reduction has great influence on the texture intensity, the orientation distribution, inclination direction and angle of the basal pole. The texture finally transforms into a gradually weakening $\{0001\}$ basal texture with highly centrally toward ND. The $\{10 \overline{1}$ $0\}$ and $\left\{\begin{array}{lll}1 & \overline{2} & 10\end{array}\right\}$ prismatic textures gradually evolve from 

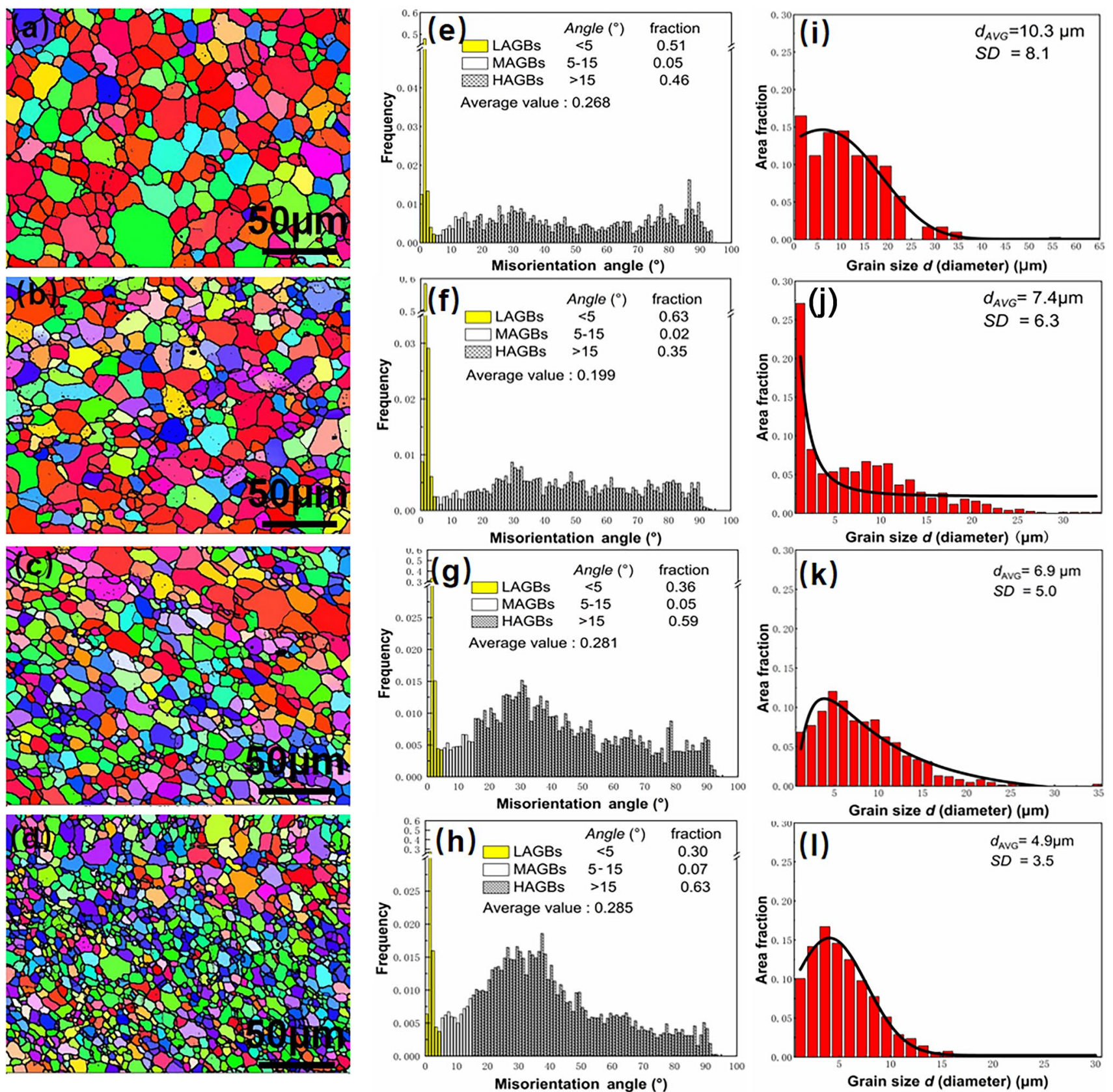

Fig. 3 Microstructure characteristics of the alloy after the multi-rolling processes: $\mathbf{a}-\mathbf{d}$ IPF maps; $\mathbf{e}-\mathbf{h}$ grain misorientation distribution maps; $\mathbf{i}-\mathbf{l}$ average grain size vs area fraction distribution maps and the black curve means lognormal fitting of the grain size distributions

higher random dispersion at no specific direction to continue distribution toward ND.

\subsection{Tensile Properties}

The typical engineering stress-strain relationships at room temperature of the extruded and multi-rolled samples are shown in Fig. 6. The yield stress (YS), ultimate tensile stress (UTS) and failure elongation (EL) are listed in
Table 3. As shown in Table 3, the mechanical properties of the extruded plate are uneven, and the deformation in the middle of the plate is less than that in other areas. In the rolling process, the ultimate tensile stress, yield stress and failure elongation all show an increasing trend. These failure elongation criteria of the $4^{\text {th }}$ rolling pass exhibited the highest value of $25.5 \%$. Moreover, the improvement in yield stress during the multi-pass rolling process can be attributed to grain refinement. 


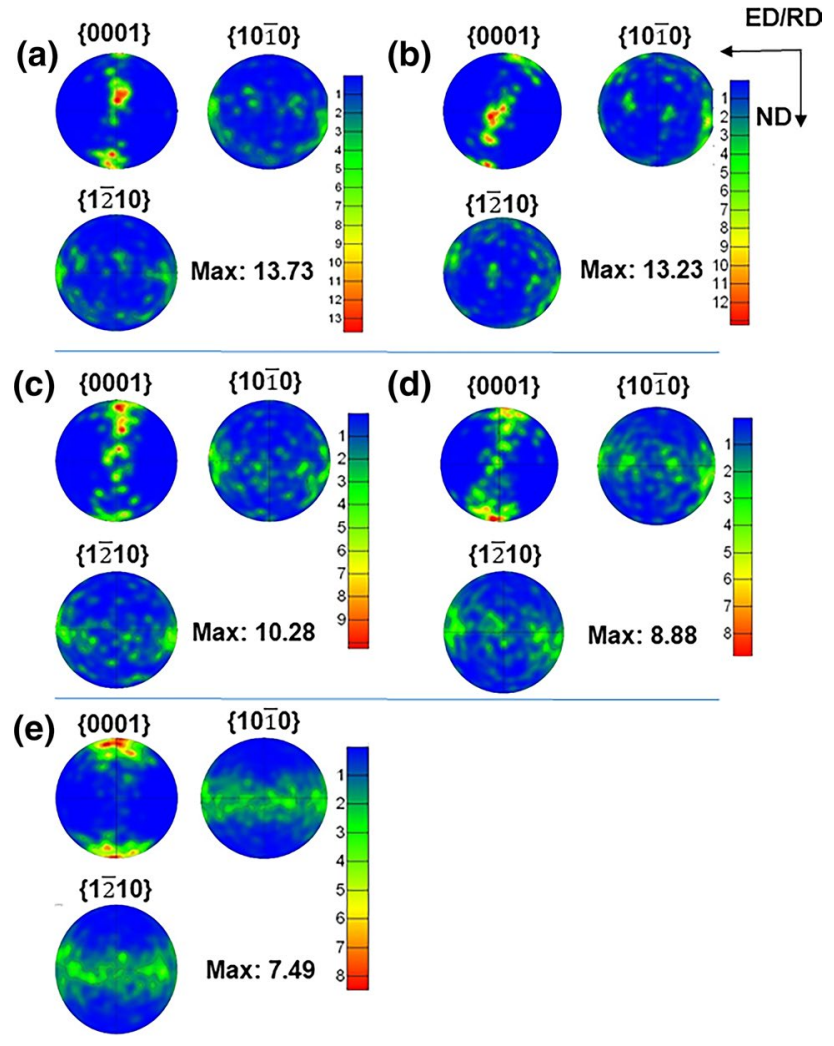

Fig. $4\{0001\},\{10 \overline{1} 0\}$ and $\{1 \overline{2} 10\}$ pole figures of the extruded and rolled samples: a the original extruded sample; $\mathbf{b}$ the rolled (pass 1) sample; $\mathbf{c}$ the rolled (pass 2) sample; $\mathbf{d}$ the rolled (pass 3) sample; $\mathbf{e}$ the rolled (pass 4) sample

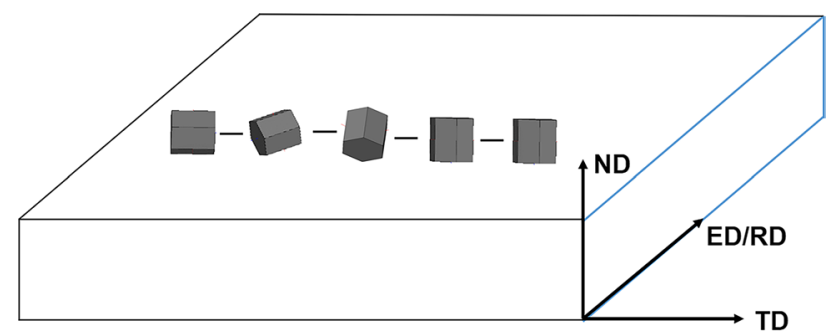

Fig. 5 Schematic diagram of grain rotation in the selected plane (ED/ RD-ND plane)

\section{Discussion}

\subsection{DRX Mechanism During Rolling Processes}

There are three nucleation modes during the rolling process which are particle-stimulated nucleation, grain boundary nucleation, and twin induced nucleation. Figure 7 shows the TEM micrographs of the DRX characteristics of the four pass rolled sample. Due to the high deformation strain, high density of dislocations and many

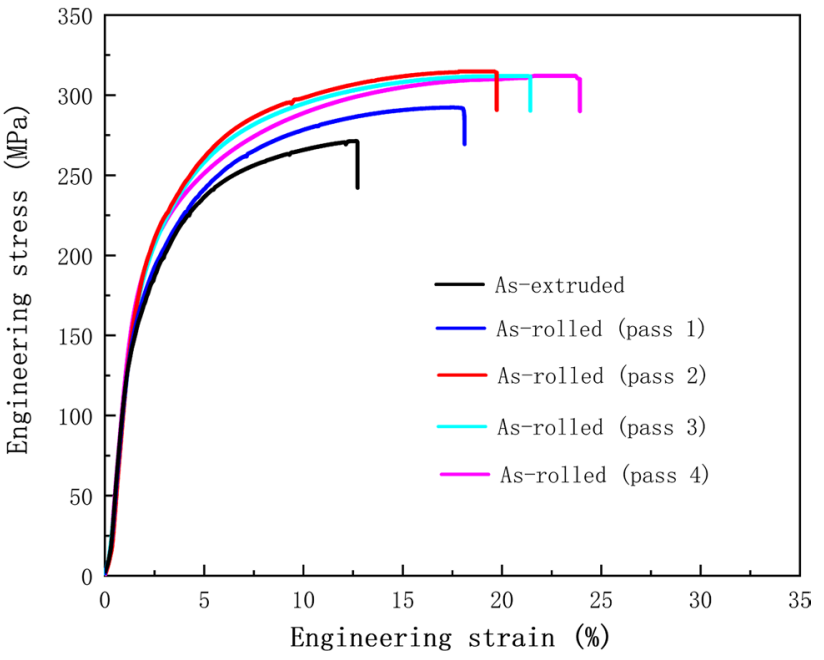

Fig. 6 Engineering stress-strain carves of the extruded and rolled samples along the RD

Table 3 Summarized mechanical properties of the AZ80RE Mg alloy sheets

\begin{tabular}{llll}
\hline Sample & UTS $(\mathrm{MPa})$ & YS $(\mathrm{MPa})$ & EL $(\%)$ \\
\hline As-extruded & $269.7 \pm 2.2$ & $141.5 \pm 1.6$ & $10.2 \pm 1.2$ \\
As-rolled (pass 1) & $291.7 \pm 1.5$ & $177.7 \pm 2.1$ & $15.6 \pm 0.9$ \\
As-rolled (pass 2) & $311.5 \pm 1.1$ & $179.2 \pm 1.8$ & $18.8 \pm 1.1$ \\
As-rolled (pass 3) & $311.2 \pm 2.0$ & $193.9 \pm 1.7$ & $21.4 \pm 0.8$ \\
As-rolled (pass 4) & $312.8 \pm 1.6$ & $196.3 \pm 1.6$ & $25.5 \pm 0.8$ \\
\hline
\end{tabular}

dislocation tangles can be seen in the microstructure. Figure 8 presents the EBSD mappings of different types of grains, which shows recrystallized grains (blue area), substructures (yellow area), and deformed grains (red area). The results reveal that the increasing strains significantly enhance the fraction of recrystallized grains. The increasing effective strain, to a certain extent, is beneficial to the introduction of higher density dislocations in the material and the restraints of dynamic recovery of dislocations and further refinement of the grain. High-density dislocation induces the formation of sub-grains which are the major nucleation points of DRXed grains [16]. The grain boundaries which form by accumulated dislocations during rolling process turn to HAGBs by rotational rearrangements [17]. Those characteristics of dislocation are due to the high degree of deformation. In Fig. 7a, high-density dislocations tangle around a second phase. The deformative difference around the particle and the matrix produces stress concentration, which obstructs deformation, produces dislocation sources, and increases the dislocation density in the matrix [18]. The unparalleled dislocation lines inter-cross during the dislocation diffusion process, 


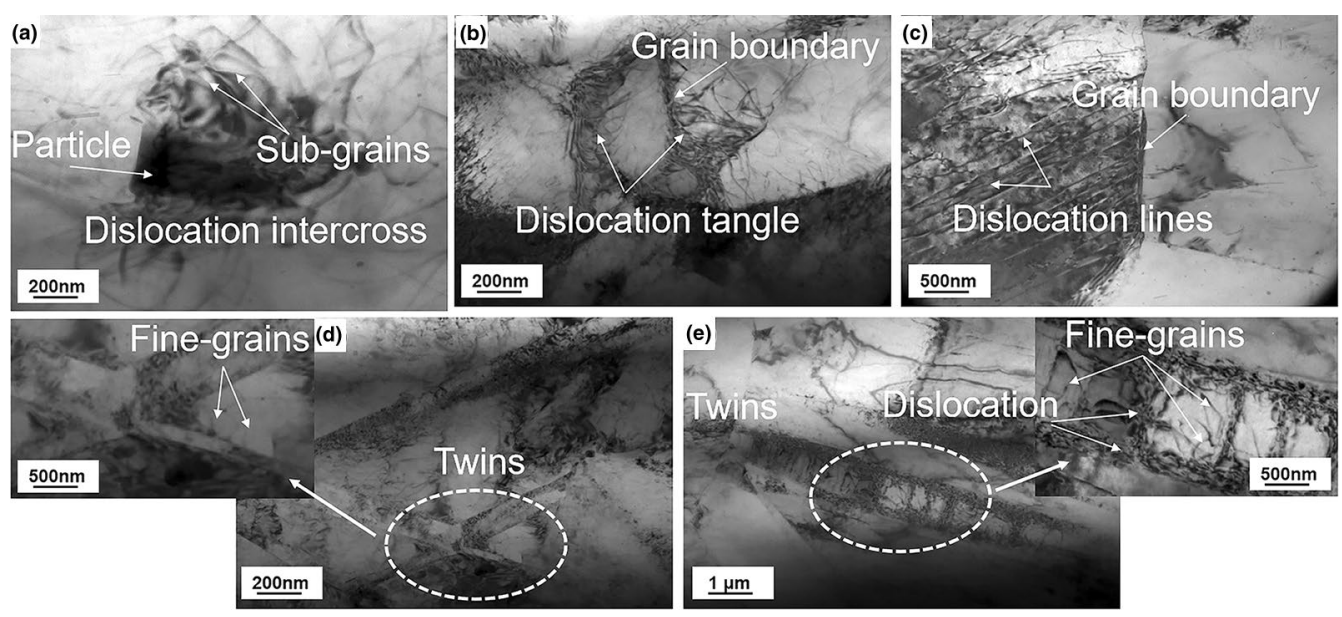

Fig. 7 TEM micrographs of nucleation characteristics of DRXed grains of the 4th rolled sample: a dislocation around the particle; $\mathbf{b}$ dislocation tangle in the grain boundaries; $\mathbf{c}$ high-density dislocations in the grain; $\mathbf{d}$ fine grains in the confluence of twins; $\mathbf{e}$ DRX nucleation in the twins
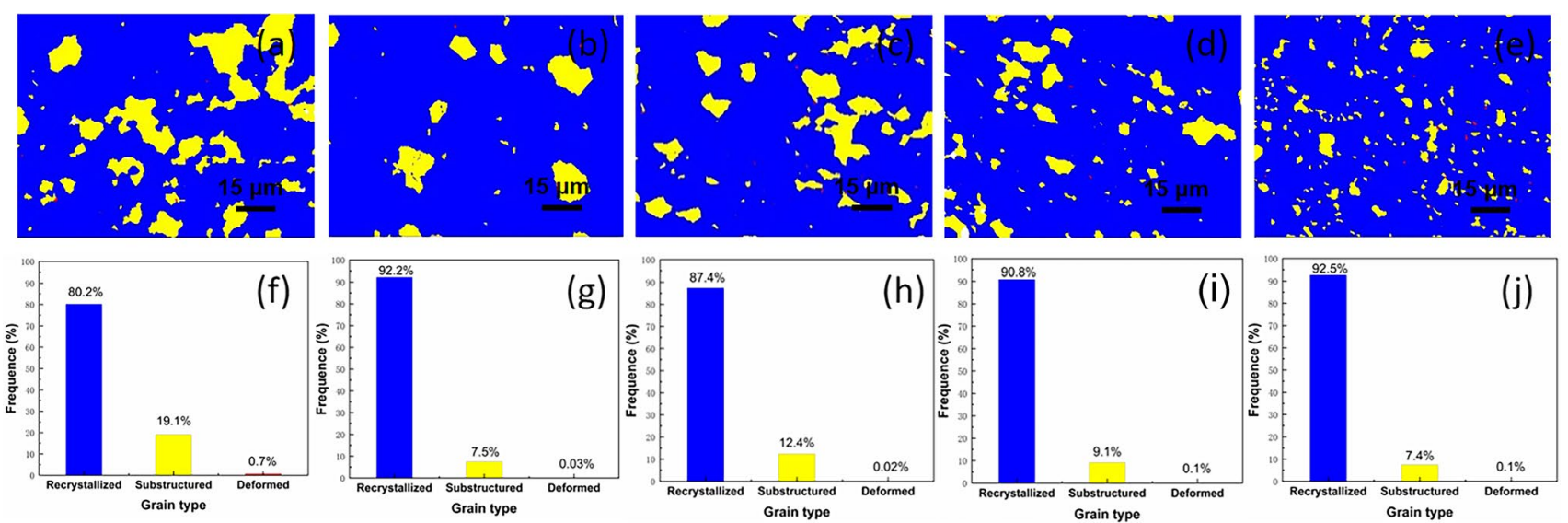

Fig. 8 Different types of grain of the sheets a as-extruded; b as-rolled (pass 1); c as-rolled (pass 2); d as-rolled (pass 3); e as-rolled (pass 4): blue-recrystallized grains, yellow—substructures, red-deformed grains; $\mathbf{f}-\mathbf{j}$ frequency of the different types of grains shown in $\mathbf{a}-\mathbf{e}$, respectively

which further evolve to dislocation cell structures. The dislocation cell structures further transform to substructures. The substructures grow up by resorting dislocations and develop into high angle grain boundaries. Figure $7 \mathrm{~b}$ demonstrates high-density dislocations tangle at the grain boundaries. Grain boundaries can impede the motion of dislocations (seen in Fig. 7c) which results in dislocation pile-up and tangling. In Fig. 7d, some fine grains emerge in the confluence of twins. In Fig. 7e, the twins boundaries evolve to dislocation walls which are divided into several dislocation rings. Some fine grains are born in those dislocation rings. Dislocation-twins interaction occurs when strain concentration occurs at the twins boundaries and within the twins [19]. Dislocation accumulation between twins boundaries and the twins can even transform the twins boundaries into HAGBs, bringing enough strain energy and preferential nucleation particles [20].

\subsection{Influence of Grain Size and \{0001\} Basal Texture on Yield Stress of the Rolled Sheets}

According to the Hall-Petch law: $\sigma_{0.2}=\sigma_{0}+k d^{-1 / 2}$, yield strength of the as-rolled sheets increases due to the greatly grain refinement. On the other hand, yield strength is inversely proportional to the average Schmid factor according to Schmid law: $\sigma_{\mathrm{s}}=\tau_{\mathrm{c}} / m$ [21]. Therefore, the enhancements of the yield stress are influenced by grain refinement and $\{0001\}$ basal texture. Figure 9 shows the relationship between engineering stress and $d^{-1 / 2}$. Grain refinement effectively strengthens the sheets via dislocation stagnation following the Hall-patch formula. Obviously, the yield stress and ultimate tensile stress are all positive associated with the value of $d^{-1 / 2}$, and the constant $k$ is a positive slope. Distinguished according to the effect of grain refinement on the yield strength, diagram of 


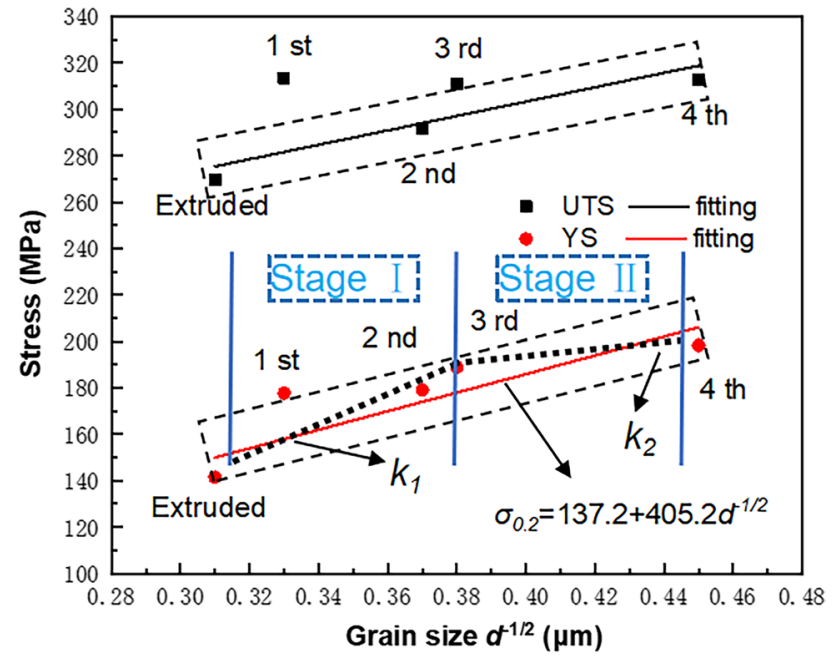

Fig. 9 UTS and YS are plotted against the $d^{-1 / 2}$ for the extruded and rolled AZ80RE alloy

different rolling pass can be divided into two stages: stage I and stage II, according to the two styles increase rate of yield stress, seen in Fig. 9. The slope of the two linear regression lines is regarded as $k_{1}$ and $k_{2}$, and the value of $k_{1}$ is obvious higher than $k_{2}$, which means the effect of grain refinement on yield strength in stage I was greater than that in stage II. Grain refinement and basal texture intensity both affect the yield stress during different rolling stage $[22,23]$. The line of the stage I shows inerratic Hall-patch relationship. It illustrates that the basal texture intensity has little influence on yield stress in this work. The decline of basal texture intensity is not enough, and the effect of the grain refinement is dominant. Normally, the decrease in yield stress after different rolling passes can be attributed to the weakening of basal texture, under the premise of grain refinement [24].

As shown in Figs. 4 and 5, during the process of multipass rolling, the texture type and strength change. The texture type is changing from rolling texture to fiber texture, and the texture strength is also decreasing. Before the 3rd pass rolling, although the texture strength decreased, the texture type remained unchanged, and the increase in yield strength was mainly due to the effect of grain refinement. The yield strengths of the last two rolled plates change little. Texture softening has a certain effect on yield strength when grain refinement reaches a certain degree. Based on the Schmid law $\sigma_{\mathrm{s}}=\tau_{\mathrm{c}} / m$, when there is preferred orientation existing in the grains, the formula is rewritten as $\sigma_{s-t}=\tau_{c-t} / m_{t}$, and when there is no influence of texture on the yield stress, the formula is $\sigma_{s-r}=\tau_{c-t} / m_{r}$. $\left(\sigma_{s}\right.$ is yield stress, $\tau_{c}$ is critically resolved shear stress which is an intrinsic constant and it is irrelevant with loading mode and crystal orientation, $m$ is Schmid factor where $m_{\mathrm{r}}$ is average Schmid factor and $m_{\mathrm{t}}$ is Schmid factor without texture effects). Therefore, the relationship between $\sigma_{\mathrm{s}}$ and Schmid factor can be obtained as $\sigma_{\mathrm{s}-\mathrm{t}}=m_{\mathrm{r}} / m_{\mathrm{t}} \sigma_{\mathrm{s}-\mathrm{r}}$. Combined with Hall-Patch relationship, the formula is rewritten as $\sigma_{\mathrm{s}-\mathrm{t}}=m_{\mathrm{r}} / m_{\mathrm{t}}\left(\sigma_{0}+k d^{-1 / 2}\right)$. The average Schmid factor $m_{\mathrm{r}}$ of the $\mathrm{Mg}$ alloy with random orientation is regarded as a constant. As a result, according to the revised HallPatch formula, grain size and texture are related to the yield stress. Figure 10 depicts the distribution maps of Schmid factor (SF) for $(0001) /<11 \overline{2} 0>$ basal ship of the four rolling sheets along the ED. The result illustrates that
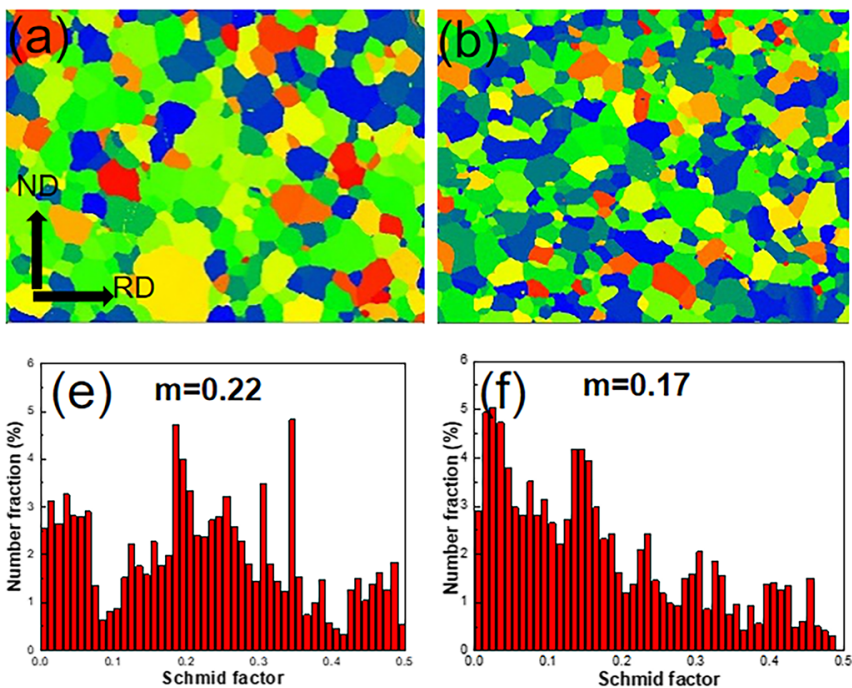
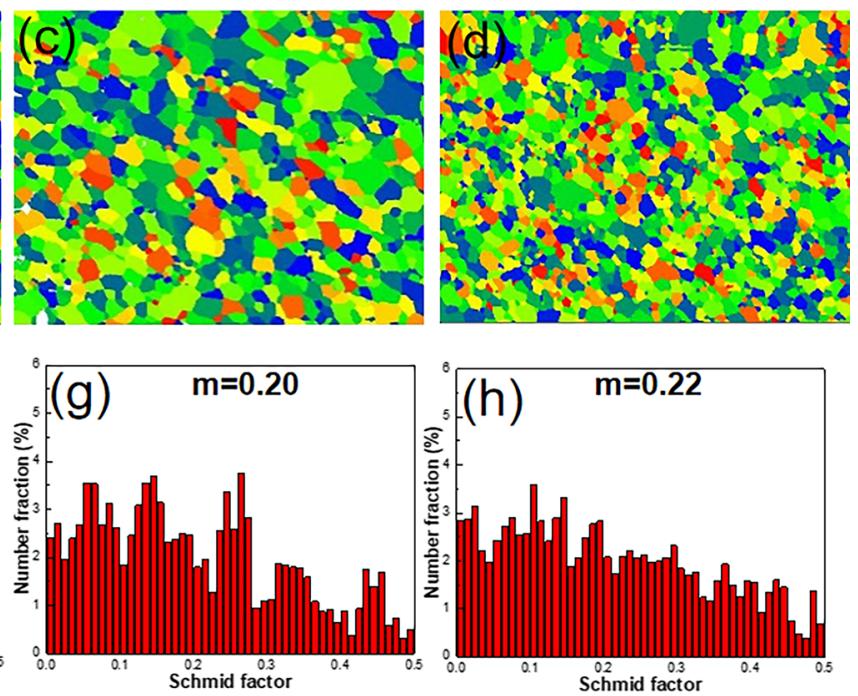

Fig. 10 Quantitative analysis of $(0001) /<11 \overline{2} 0>$ basal ship Schmid factor (SF) of the multi-rolling samples: a, e as-rolled (pass 1); b, f asrolled (pass 2); c, $\mathbf{g}$ as-rolled (pass 3); $\mathbf{d}, \mathbf{h}$ as-rolled (pass 4) 


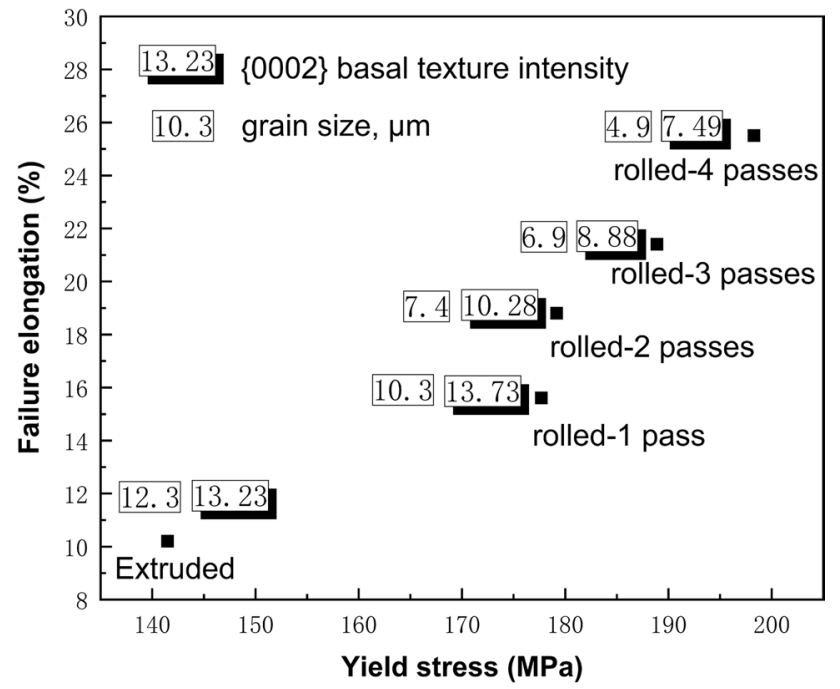

Fig. 11 Variations failure elongation of the AZ80RE alloy sheets during extrusion and multi-rolling processes as a function of the correlated yield strength

increase in rolling reduction can significantly increase the $\mathrm{SF}$ for $(0001) /<11 \overline{2} 0>$ basal ship which means the easier activation of basal ship.

\subsection{Influence of Grain Size and \{0001\} Basal Texture on the Ductility of the Rolling Sheets}

Figure 11 shows variations failure elongation of AZ80RE alloy sheets during extrusion and multi-rolling processes along the RD as a function of the correlated yield stress. The grain size and $\{0001\}$ basal texture intensity are also marked on the figure. The results illustrate that the failure elongation of the sheets depends on grain size and $\{0001\}$ basal texture during the deformation process. The grain size ranges from $4.9 \mu \mathrm{m}$ to $12.3 \mu \mathrm{m}$, and the $\{0001\}$ basal texture intensity ranges from 7.49 to 13.73. As for ductility, the grain size displays remarkable effects. The decrease in the grain size can exhibit larger elongation. It is suggested that more slip system could be activated in the finer grain size and the dislocation glide dominated plasticity may have grain size dependence [23]. The research of W.Z. Chen et al. [25] shows that the ductility enhance in $\mathrm{Mg}$ alloy as the weakening of $\{0002\}$ basal texture intensity in a wider grain size of $2.5-12 \mu \mathrm{m}$. The finer-grains with relatively weak basal texture display randomicity of grain orientation attributed to the continuous dynamic recrystallization (CDRX). Additionally, it is hard to ascertain which pattern is more effective to improve the ductility of the AZ80RE alloy in this work. However, it is no doubt that remarkable grain refinement and lower basal texture should be considered in the rolling processes.
The evolution of initial texture intensity, the orientation distribution and the inclination direction of the basal pole mainly attribute to by DRX mechanism and slip mechanism $[24,25]$. It was reported that most of these coarse grains dominate initial strong basal texture after DRX $[26,27]$. With the increase in reduction during per pass rolling process, the DRXed mode transfers to CDRX and the coarse grains annihilate by more fine grains. The fine grains are rotated more randomly, which are not favorably orientated to the basal plane with the c-axis parallel to $\mathrm{ND}$, resulting in the weakening of the base plane texture. In general, the tilt of $\{0001\}$ basal pole is usually related to the sliding mechanism. The visible inclination of the basal pole mainly due to the prismatic $<\mathrm{c}+\mathrm{a}>$ slip and some pyramid $<\mathrm{c}+\mathrm{a}>$ slip. The increasing rolling pass can activate more pattern of slip system which facilitates the further deformation.

\section{Conclusions}

Fine-grained AZ80RE Mg alloy with enhanced ductility was established after extrusion and multi-rolling processes. The microstructure, mechanical properties and texture evolution of the extruded and rolled sheets were investigated in this work. The specific conclusions are as follows:

1. The rolling process has significant effect on grain refinement of the extruded plate. With the increase in rolling pass, the proportion of DRXed grains increase due to particle-stimulated nucleation, grain boundary nucleation and twin induced nucleation.

2. The $\{0001\}$ basal pole gradually titles from TD to ND during multi-rolling process because of the asymmetric deformation and irregular grain deflection which results in weakening basal texture intensity. The evolution of initial texture intensity, orientation distribution and inclination direction of the basal pole attribute to DRX mechanism and slip mechanism.

3. Grain refinement and basal texture intensity have combined effect on the mechanical properties. Grain refinement is the main reason for the increase in yield stress. The finer-grains with relatively weak basal texture display randomicity of grain orientation, which resulted in a remarkable ductility.

Acknowledgements This research was financially supported by the National Key Research and Development Program of China (Grant No. 2016YFB0301104) and the National Natural Science Foundation of China (Grant No. 51771043). The authors are also grateful for the support from the State Key Laboratory of Baiyunobo Rare Earth Resource Researches and Comprehensive Utilization. 


\section{References}

[1] Q.Y. Liao, X.R. Chen, Q. Lan, F.K. Ning, Q.C. Le, Mater. Res. Express 5, 126510 (2018)

[2] Y.P. Ding, Q.C. Le, Z.Q. Zhang, L. Bao, J. Cao, J.Z. Cui, J. Mater. Process. Technol. 213, 2101 (2013)

[3] X. Li, T.A. Samman, G. Gottstein, Mater. Des. 32, 4385 (2011)

[4] Q. Miao, L.X. Hu, G.J. Wang, E. Wang, Mater. Sci. Eng., A 528, $6694(2011)$

[5] W.K. Wang, W.Z. Chen, W.C. Zhang, G.R. Cui, E. Wang, Mater. Sci. Eng., A 712, 608 (2018)

[6] M.G. Zelin, H.S. Yang, R.Z. Valiev, A.K. Mukherjee, Metall. Mater. Trans. A 23, 3135 (1992)

[7] M.Y. Zhan, C.M. Li, W.W. Zhang, D.T. Zhang, Acta Metall. Sin. -Engl. Lett. 25, 65 (2012)

[8] A. Imandoust, C.D. Barrett, A.L. Oppedal, W.R. Whittington, Y. Paudel, H.E. Kadiri, Acta Mater. 138, 27 (2017)

[9] D.K. Guan, W.M. Rainforth, J.H. Gao, L. Ma, B. Wynne, Acta Mater. 145, 399 (2018)

[10] Y.M. Kim, C. Mendis, T. Sasaski, D. Letzig, F. Pyzak, K. Hono, S. Yi, Scr. Mater. 136, 41 (2017)

[11] M. Muzyk, Z. Pakiela, K.J. Kurzydlowski, Scr. Mater. 66, 219 (2011)

[12] J.H. Zhang, D.P. Zhang, T. Zheng, J. Wang, K. Liu, H.Y. Lu, D.X. Tang, J. Meng, Mater. Sci. Eng., A 489, 113 (2008)

[13] A. Gledhill, R. Kaibyshev, G. Gottstein, Acta Mater. 49, 1199 (2001)
[14] G. Zeng, C.M. Liu, Y.H. Gao, S.N. Jiang, Mater. Sci. Eng., A 674, 491 (2016)

[15] X.S. Huang, S. Kazutaka, S. Ichinori, S. Naobumi, Scr. Mater. 60, 964 (2009)

[16] H.D. Fan, S. Aubry, A. Arsenlis, J.A. Awady, Acta Mater. 92, 126 (2015)

[17] C. Xu, M.Y. Zheng, S.W. Xu, K. Wu, E. Wang, G.H. Fan, S. Kamado, Mater. Sci. Eng., A 643, 137 (2015)

[18] H.C. Pan, G.W. Qin, Y.M. Huang, Y.P. Ren, X.C. Sha, X.D. Han, Z.Q. Liu, C.F. Li, X.L. Wu, H.W. Chen, C. He, L.J. Chai, Y.Z. Wang, J.F. Nie, Acta Mater. 49, 350 (2018)

[19] T.T. Sasaki, K. Yamamoto, T. Honma, S. Kamado, K. Hono, Scr. Mater. 59, 1111 (2008)

[20] H.X. Zhang, S.F. Chen, M.C.C. Zheng, S.H. Zhang, Acta Metall. Sin. Engl Lett 32, 1122 (2019)

[21] D. Liu, Z.Y. Liu, E. Wang, Mater. Sci. Eng., A 612, 208 (2014)

[22] L.L. Guo, Z.M. Chen, L. Gao, Mater. Sci. Eng., A 528, 8537 (2011)

[23] Y.P. Wu, X.M. Zhang, Y.L. Deng, C.P. Tang, Y.Y. Zhong, Mater. Sci. Eng., A 616, 148 (2014)

[24] W.Z. Chen, X. Wang, E.D. Wang, Z.Y. Liu, L.X. Hu, Scr. Mater. 67, 858 (2012)

[25] X. Li, W. Qi, Mater. Sci. Eng., A 560, 321 (2013)

[26] J.A.D. Valle, M.T. Pérez-Prado, O.A. Ruano, Mater. Sci. Eng., A 355, 68 (2003)

[27] A. Yamashita, Z. Horita, T.G. Langdon, Mater. Sci. Eng., A 300, $142(2001)$ 